ROSAT

\title{
A NEW LOOK AT THE SKY
}

\author{
J.E. Trümper \\ Max-Planck-Institut für Extraterrestrische Physik \\ Garching, Germany
}

X-ray astronomy, which in the last three decades has become one of the main tools for exploring the Universe, is presently receiving fresh stimulus from the ROSAT satellite observatory launched on 1 June 1990. The list of known cosmic X-ray sources includes almost all kinds of astronomical objects, ranging from the Sun and nearby stars out to quasars at the edge of the known Universe. In many sources, the $\mathrm{X}$-rays are emitted by plasmas with temperatures of millions to billions of degrees - conditions found in stellar corona, in shock regions produced by strong stellar winds or supernova explosions, in accretion disks around compact objects, or in the intergalactic gas in clusters of galaxies.

Another important emission mechanism is synchrotron radiation by ultra-relativistic electrons as seen in the Crab nebula and other supernova remnants containing active pulsars. Inverse Compton scattering of relativistic electrons with photons plays a rôle in other sources. Whatever the mechanism, the emission of $\mathrm{X}$-rays requires extreme conditions in the source region and is often connected with explosive events.

ROSAT (Fig. 1) carries a large imaging $\mathrm{X}$-ray telescope working in the 0.1-2.4 keV energy range and providing a 3-10 fold improvement in angular resolution and sensitivity in the pointing mode compared to predecessors, namely NASA's Einstein Observatory and ESA's EXOSAT. A novel feature is its capability to make images in 4-5 colour bands over the 0.1-2.4 keV energy range. There is also a smaller XUV telescope working at $40-200 \mathrm{eV}$. The satellite involves an international collaboration in which Leicester University, leading a UK consortium, suppled the wide-field camera for the XUV telescope. The satellite was launched by NASA and the Smithsonian Astrophysical Observatory supplied the high resolution imager for the X-ray telescope. Germany supplied the satellite, overall project management, the main ground station, the $\mathrm{X}$-ray mirrors, and the remaining hardware items.

Professor J.E. Trümper has been Director of the MPI für Extraterrestrische Physik, W-8046 Garching bei München, Germany, since 1975. $\mathrm{He}$ studied at Universities in Halle, Hamburg, and Kiel where he held academic posts before moving to direct the University of Tübingen's Astronomical Inst. in 1971 as Full Professor.

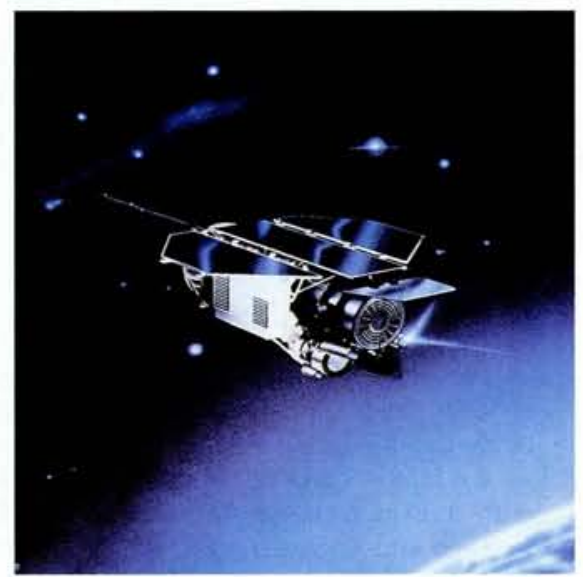

Fig. 1 - The ROSAT in orbit (artist's impression). The $X$-ray telescope comprises a $84 \mathrm{~mm}$ in diameter 4-fold nested Wolter 1 system with two position-sensitive proportional counters $10.1-2.4 \mathrm{keV}, 2^{\circ}$ field of view, 25 arcsec resolution, 42\% FWHM energy resolution) and a high resolution multichannel plate detector $10.1-2.4 \mathrm{keV}$, $0.6^{\circ}, 4$ arcsec). The XUV telescope comprises a $57 \mathrm{~mm}$ in diameter 3-fold nested Wolter-Schwarzschild system with 2 microchannel plate detectors $140-200 \mathrm{eV}, 5^{\circ}$, 1 arcmin) with filter spectroscopy in two bands.

\section{All-Sky Survey and}

\section{Detailed Observations}

A major and novel task ROSAT performed was the first all-sky survey with an imaging telescope. The hitherto most sensitive survey of this type, made with the HEAO-1 satellite in $1977 / 8$, resulted in 840 sources. About 60000 sources have being found in the ROSAT survey (Fig. 2), which has been completed but is not yet fully analysed. This represents a wealth of new information on individual sources, and opens up the possibility of source/ class studies based on large, unbiased samples.

Following the sky survey which took six months, ROSAT is now being used to carry out detailed pointing observations of individual sources - a programme which is open to guest observers. A large number $(1500)$ of proposals were submitted for the first year, exceeding the available time by a factor of six and demonstrating the strong interest of a widely distributed international community in X-ray telescope observations.
In the following, we shall summarise some of the early scientific highlights of ROSAT's mission.

\section{Sun, Moon and Stars}

Lunar scattering and bremsstrahlung

The birth of cosmic X-ray astronomy in 1962 was accidental. The rocket experiment of Giacconi et al. aimed to detect $X$-rays from the Moon which were expected from the scattering of solar X-rays. Instead, the group found something much more interesting, namely Sco $X-1$, the strongest $\mathrm{X}$-ray source in the sky, and a diffuse sky background of $X$-rays.

Detection of soft lunar $\mathrm{X}$-rays from an Earth-orbiting satellite was in fact accomplished nearly 28 years later in an early ROSAT observation. Fig. 3 shows the sunlit crescent on the Moon, thus demonstrating that the lunar $\mathrm{X}$-ray luminosity arises

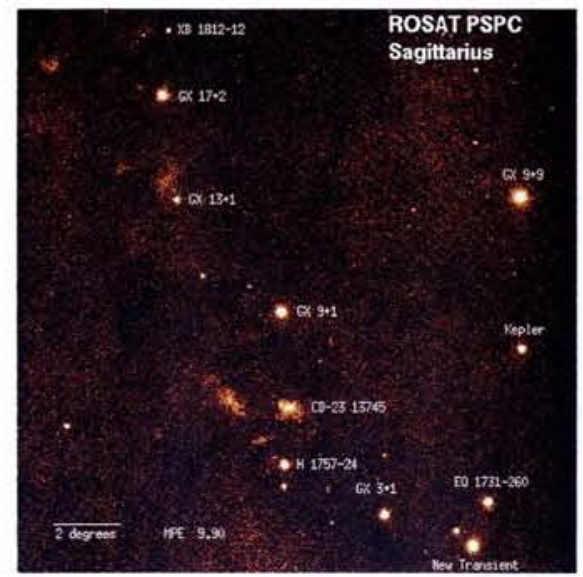

Fig. 2 - A section of the ROSAT all-sky survey close to the galactic centre.

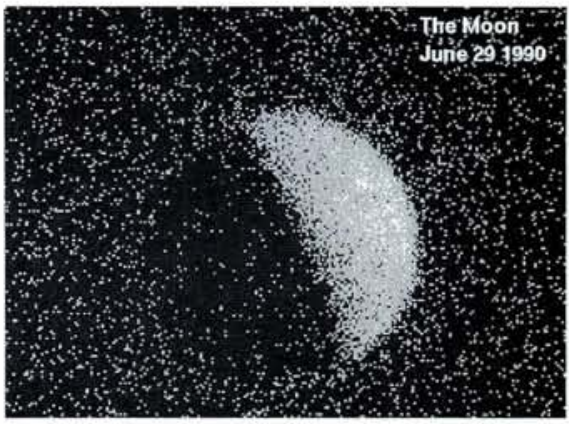

Fig. 3 - The first $X$-ray image of the Moon taken in the soft $X$-ray band shows the crescent as well as a distinct $X$-ray shadow in the diffuse $X$-ray background cast by the dark side of the moon. 


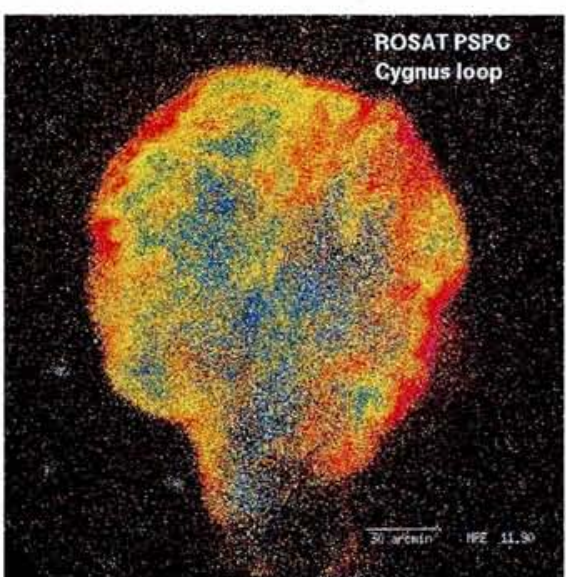

Fig. 4 - A ROSAT image of the Cygnus loop, an ancient supernova remnant seen in detail for the first time. The colour codings denote the average photon energy per pixel (red: low; blue: high).

from the backscattering of solar coronal $X$-rays. The Moon casts a distinct shadow in the diffuse cosmic X-ray background. A real surprise was the discovery of a low level X-ray flux from the dark side of the Moon: this may be due to bremsstrahlung from magnetic solar wind electrons striking the lunar surface [1].

\section{Orbiting white dwarfs}

Cool stars possess hot X-ray emitting coronae which are very likely structured and heated by magnetic fields. Hot stars are thought to produce $\mathrm{X}$-rays by means of their strong, radiatively driven winds which become unstable and dissipate prodigious amounts of energy in shocks. With 15-20000 stellar X-ray sources, the ROSAT survey provides a very large statistical basis for correlations of $\mathrm{X}$-ray fluxes and temperatures with spectral types and classes, magnetic fields and rotational velocities.

Unexpectedly, ROSAT detected X-ray emission from a number of A-stars which were supposed to be $\mathrm{X}$-ray quiet, being located between coronal- and stellar-wind type sources. In the meantime, there is strong evidence that, at least in some of these systems (e.g. Beta Crateris [2]), the emission comes from a white dwarf orbiting the A-star. The detection of such Sirius-type systems is of great interest because it should be possible to estimate the mass of the white dwarf, thus improving our understanding of the small number of such objects for which the mass is known.

\section{Red giants absorb}

Another surprise was that the number of single white dwarfs detected in the survey is an order of magnitude smaller than the pre-ROSAT estimate. This is probably due to photoelectric absorption by heavier elements in the photospheres about which nothing was known before.
One of the great puzzles concerning stellar X-rays has been the lack of X-ray emission from red giants. ROSAT observations deep into space have pushed down the upper flux limits considerably. For Arcturus, the coronal flux density at the stellar surface must be less than $10^{-4}$ times smaller than that for the Sun [Aires T.R. et al., Astrophys. J., submitted].

\section{Many more accreting objects}

ROSAT has also increased the number of known binary systems containing matter accreting white dwarfs, neutron stars or black holes. These systems are extremely bright and their observation has been a notable feature of X-ray astronomy since the early days. ROSAT will give more detailed information about the soft emission which is often radiation which has been reprocessed (scattering; heating and re-emission). The high sensitivity of ROSAT also allows the detection of accreting objects in nearby galaxies such as the Large and Small Magellanic Clouds. For example, about 180 sources have been found in the Andromeda nebula, of which about 25 could be identified with X-ray binaries, most of them residing in globular clusters.

\section{Supernova Remnants and \\ Diffuse Galactic Emission}

The remnants of the giant stellar explosions marking the end of massive stars are the most beautiful objects in the X-ray sky. ROSAT in its sky survey detected almost all of the approximately 50 known X-ray supernova remnants. Fig. 4 shows the Cygnus loop as an example which could be imaged completely and in detail for the first time owing to the "unlimited field of view" of the all-sky survey.

\section{Pressure variations found}

The other new aspect is that ROSAT obtains colour images. In the absence of interstellar absorption, the colour relates directly to the temperature $T$ of the emitting gas ("colour temperature"). At the same time, the density $n$ of the radiating plasma can be estimated from the observed brightness $\left(\sim n^{2}\right)$, leading to the possibility of estimating the thermal pressure $(n \times T)$ distribution over the remnant. Surprisingly, pressure differences of up to a factor of 50 are found within one supernova remnant whereas one expected conditions equilibrium: the reasons for such large deviations from equilibrium are not yet understood [Aschenbach B., private communication].

The sky survey also brought a rich harvest of new supernova remnants (about 50 to 60 ) which had not been seen before in the radio and optical bands. The first example was a shell source of almost $2^{\circ}$ in diameter in Auriga which probably exploded in a low density region of the interstellar medium [3].

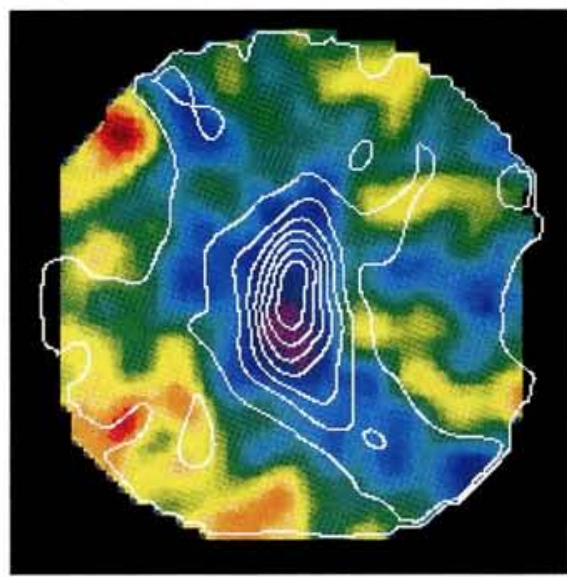

Fig. 5 - The coloured diagram shows a shadow in the diffuse $X$-ray flux distribution cast by photoelectric absorption of the cloud (cloud contour lines measured by the IR satellite IRAS at $100 \mu \mathrm{m}$ ).

\section{Supernova 1987A: disappointing}

Disappointing, but nevertheless interesting, were the observations of the Supernova 1987A explosion. We considered this object to be important enough to make it ROSAT's "first light" target. The first and all subsequent observations yielded a series of decreasing upper limits showing that the thermal emission from the supernova's blast wave is not yet strong enough to generate $\mathrm{X}$-rays, and that the view to the central (probably hot) region is still obscured by the expanding cloud [4].

\section{The first shadows from cool clouds}

The hot phase of the interstellar medium has a temperature of about $10^{6} \mathrm{~K}$ and is believed to be the result of many old supernova remnants which have been dissolved, leading to a patchy, diffuse X-ray emission of low surface brightness. This diffuse emission could be imaged directly for the first time owing to the low intrinsic background of the ROSAT detector. The sky survey thus contains much information about the distribution of hot plasma and of the cool gas which absorbs X-rays.

Of special interest in this context is the discovery of the first X-ray "shadows" cast by cool interstellar clouds [Snowden S. et al., Science, in press; Burrows D.N. et al., Nature, submitted]. This allows one to separate "foreground" and "background" emissions originating in front of and behind the absorbing cloud. Fig. 5 shows one of these shadows.

However, a large number of such measurements is necessary in order to derive a coherent picture of the large scale distribution of the hot plasma in our Galaxy. The important open question is whether a static galactic "halo" or an outflow of hot plasma exists.

Diffuse emissions and absorption patterns are also found in the $\mathrm{X}$-ray images of nearby galaxies, in particular the Large and Small Magellanic Clouds [4]. 


\section{Interstellar dust}

Another important aspect is the measurement of $\mathrm{X}$-ray halos which surround point sources because of scattering on interstellar dust grains. Similar observations to those performed on bright sources by previous instruments can be carried out with improved sensitivity using ROSAT because of the large collecting power and the extremely low microroughness ( $3.5 \AA \mathrm{RMS}$ ) of the mirror system. First results [5] show that the grain size distribution in the range $0.04-0.4 \mu \mathrm{m}$ is measured much more accurately.

\section{Galaxies, Clusters of Galaxis and Quasars}

It is well known that galaxies are not distributed randomly in space but show distinct clustering effects. Clusters of galaxies are strong $\mathrm{X}$-ray emitters and their radiation comes from a hot plasma produced by galaxy-galaxy collisions. While not too much happens to the stars in such events, it would appear that the interstellar media of the colliding galaxies are shock heated and swept out into intergalactic space.

Estimates show that the total number of clusters of galaxies expected to be found in the ROSAT survey is $4000-6000$ - many more than have been identified up to now using $\mathrm{X}$-rays [Böhringer $\mathrm{H}$., private communication]. Optical spectroscopic redshift measurements are scheduled and these will permit measurements of the cluster distribution out to redshifts $z$ of 0.3 or more - much further than by simply observing the distribution of galaxies $(z<0.1)$. We note also that generally well-know large scale structures such as the "Great Wall" and the "Great Attractor" are both within the reach of ROSAT's spectroscopic capabilities.

\section{Clusters grow by merging}

The main unanswered question concerning the distribution of space objects is how the initially homogeneous Universe evolved into the present highly structured state containing galaxies, clusters and superclusters. While the analysis of the large ROSAT samples will take some time, clues to the evolution of individual clusters of galaxies can be found by studying their morphology.

As a matter of fact, the ROSAT observations show that a number of bright clusters contain more internal structure than previously thought. For instance, in Abell 2256 one sees (Fig. 6) a clear double peak distribution suggesting the infall of a smaller cluster into a larger one [6]. Furthermore, the well-known Coma and Perseus clusters exhibit interesting sub-structures. It appears that one is seeing here some indications of how clusters grow, namely by "merging" or the infall of small groups of galaxies.

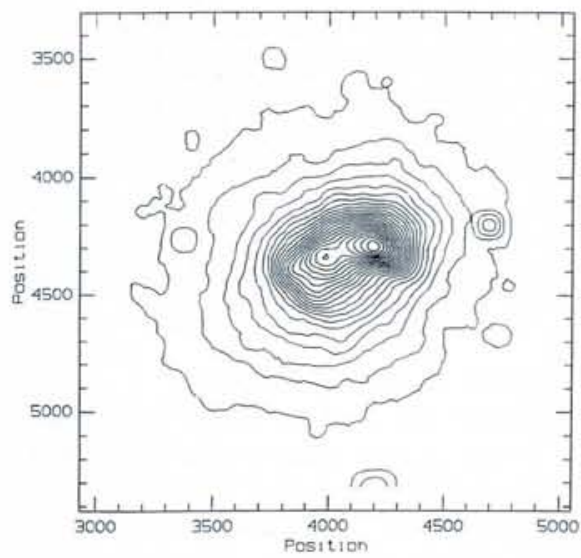

Fig. 6 - A ROSAT image of the Abell 2256 galaxy cluster reveals a clear double peak structure indicating the merging of two clusters.

\section{Feeding active galactic nuclei}

\section{or reprocessing}

Quasars and other active galactic nuclei (AGN), which represent the largest class of ROSAT survey sources (numbering about 20000), are found at even larger distances and redshifts. The few hundred of these sources which have been identified so far show redshifts of up to $z=3.8$. The ROSAT data confirm that the power law spectra of these objects generally steepens towards low energies, strongly reinforcing the evidence that these soft "X-ray excesses" and the UV excesses ("UV bumps") in AGN spectra have the same origin, i.e., they both result from a broad maximum in the XUV whose main part is obscured by interstellar photoelectric absorption. This XUV bump could be due to either emission from an accreting disk feeding a central supermassive black hole or to reprocessing.

The galactic background: a decreasing quasar density and a supercluster

The nature of the extragalatic X-ray background is still unresolved 29 years after its discovery. Is it made up from the superposition of mainly faint sources, or is it partly due to truly diffuse emission from intergalactic space? One needs to perform very deep observations to answer these questions. The longest ROSAT telescope pointing extends to flux levels almost a factor of 10 smaller than those of the Einstein Observatory: about $45 \%$ of the total extragalactic flux can be resolved into point sources [Hasinger G. et al., in preparation] which are mainly quasars at large redshifts [7]. In the deepest ROSAT field, the density of objects is 200 per square degree, i.e., more than the quasar density found in deep optical fields [Hasinger G. et al., in preparation].

The number - flux relationship $(\log N$ $\log S)$ at these weak fluxes shows a clear flattening, indicating that the space density of X-ray emitting quasars decreases beyond a redshift of $z=2$. Also of great interest is the discovery of some patchy structures in the diffuse sky background near the North Ecliptic Pole [8]. Optical observations show that these structures are due to a supercluster - the first to be discovered by $\mathrm{X}$-ray observations [Burg $\mathrm{R}$. et al., Nature, submitted].

All-in-all, it is evident that ROSAT will tell us much more about the large scale distribution of matter in our Universe.

\section{REFERENCES}

[1] Schmitt J.H.M.M. et al., Nature 349 (1991) 583.

[2] Fleming T.A. et al., Astron. \& Astrophys. Lett. 246 (1991) L47.

[3] Pfeffermann E. et al., Astron. \& Astrophys. Lett. 246 (1991) L28.

[4] Trümper J. et al., Nature 349 (1991) 579. [5] Predehl P. et al., Astron. \& Astrophys. Lett. 246 (1991) L21.

[6] Briel U. et al., Astron. \& Astrophys. Lett. 246 (1991) L10.

[7] Shanks T. et al., Nature 353 (1991) 315. [8] Hasinger G. et al., Astron. \& Astrophys. Lett. 246 (1991) L2.

\section{Complementary EC Rôle}

A Communication by the Commission of the European Community (CEC) to the EC Council and Parliament in 1998 sought to develop a systematic approach to EC space activities. For science, there resulted a series of initiatives such as an opening up of EC programmes to microgravity experiments from 1990, studies of earth observation systems, and the development of a working relationship with ESA via joint working groups.

Technical and political changes called for a fresh appraisal so Roy Gibson, ESA's former Director-General who heads a task force to set up the EC's European Environmental Agency, led a review of EC space activities by an expert panel. The panel's report The European Community: Crossroads in Space published in October called for a long-term strategy with the environment as a priority in order to intensify involvement in space-related areas. Such a strategy may emerge once an EC Parliament report updating the 1987 Toksvig Report, a second CEC Communication due in March 1992 and the results of CEC internal studies are presented.

In seeking a truly collaborative European space policy of maximum benefit building on some impressive recent achievements, the panel felt long-term EC goals should be to foster: a competitive space industry; an independent launch capability; a framework for exploiting space technology especially in telecommunications and earth observation; basic knowledge and further technologies essential for a vigorous space effort; and international collaboration. Space science was seen as a "pathfinder" with wide implications and challenging but stimulating technical requirements, so it is essential to study the Earth and the Universe in a complementary way to ESA and large multinational programmes. Space science should not be "put into a cupboard" and space scientists ought to be further encouraged to turn to EC programmes for support. 